\title{
Pode dançar provocar dor no joelho?
}

\author{
N orberto Peña ${ }^{1}$ \\ M árcia M attar ${ }^{2}$ \\ Carla Leite ${ }^{3}$ \\ Joana Lima ${ }^{3}$ \\ JamilleTeixeira ${ }^{3}$
}

\begin{abstract}
Resumo
A dor no joelho e uma das queixas mais freqüentes entre dançarinos. 0 objetivo deste trabalho foi estabelecer a prevalência e a incidência cumulativa de dor no joel ho para melhor entender a relação entre dançar e o corpo do dançarino. Constituíram a amostra 47 alunos da Escola de D ança da U FBA. Para estabelecer a freqüência de dor no joelho foram escolhidos dois desenhos metodológicos: um estudo prospectivo, para estabelecer a prevalência, eum estudo de coorte, para verificar a incidência cumulativa da variável. Como resultado, verificou-sequea prevalência de dor no joelho entre 47 alunos foi de $31.9 \%$ (15 alunos). A incidência representada manteve-seentre $13.3 \%$ e $2.2 \%$, apresentando uma gradativatendênciaà diminuição.
\end{abstract}

Palavras-chave dança; dor; joelho; incidência.

\section{INT RO DUÇÃO}

As queixas ou incômodos, inerentes ao corpo de quem dança têm sido objeto de interesse por parte das pessoas que trabalham com dança e entre os dançarinos (ASKLING et al., 2002; BROW N; MICHELI, 2004; HAMILTON; ARON SEN; LOKEN, 2006; HILLIER et al., 2004; MIKKELSSON et al., 2006). As pesquisas produzidas da observação desta população nos alertam quanto à freqüência dos problemas e localização das regiões anatômicas comprometidas (CAIN E; N ASSAR, 2005; WAIN WRIGH T; WILLIAM S; TURNER, 2005; TO LED 0 et al., 2004).

$D$ ados epidemiológicos de alunos da Escola de $D$ ança da UFBA (PEÑ A; LEITE, 1999,
2004; LEITE, 2007) revelaram que, em uma amostra de 144 alunos 0 82\% já tinham sofrido lesões e as relacionavam com a prática da dança, apresentando maior freqüência no tronco, na região lombar (47\%) e nos membros inferiores, motivo deste estudo, na região do joe Iho, com $27 \%$ dos casos.

Fora do âmbito da Escola de Dança da UFBA, estudos que relacionam etiologia, prevalência, risco e alternativas terapêuticas dão significância ao problema da dor no joelho entre dançarinos e atletas (BOZKURT et al., 2004; CIBULKA et al., 2005; CHRIST O FO RAKIS et al., 2005; FREDERICSON et al., 2006; GERBIN 0 et al., 2006; KHAUND;

\footnotetext{
${ }^{1}$ Professor da Escola de D ança - UFBA; D outor em Kinesiologia e Fisiatria da U niversidade de Buenos Aires.

2 M édica Fisiatra do Instituto N acional de Traumato O rtopedia (INTO), Rio de Janeiro.

${ }^{3}$ Alunas da G raduação da Escola de D ança da UFBA.

Correspondência para / Correppandence ta

Av. Ademar de Barros, $s / n$ - Campus de O ndina.

40.170-110 Salvador- Bahia-Brasil.

Tel.: (71) 3283-5781

E-mail: norbertopena@terra.com.br
} 
FLYNN， 2005; LABELLA， 2004; PARAD OWSKI et al., 2006; POST, 2005).

0 objetivo deste trabalho foi estabelecer a prevalência e a incidência cumulativa de dor no joelho para melhor entender a relação entre dançar e o corpo do dançarino.

\section{MATERIAIS E MÉTODOS}

A amostra foi extraída dentre os alunos matriculados na Escola de D ança da UFBA no semestre 2006.1. A participação no estudo foi voluntária e, portanto, os resultados não podem ser generalizados.

Para se estabelecer a freqüência de dor no joelho, foram escolhidos dois desenhos metodológicos: um estudo prospectivo, para estabelecer a prevalência, e um estudo de coorte, para verificar a incidência cumulativa da variável.

Do estudo de prevalência participaram 47 alunos, e uma coorte deles, composta por 32 alunos livres da condição clínica, foi acompanhada durante três meses. Os participantes apresentavam uma média de 21,34 anos (desvio padrão de 3,12 ), com um peso médio de $59.47 \mathrm{~kg}$ e uma media de altura de $167 \mathrm{~cm}$. A distribuição das idades em relação ao sexo apresentou uma idade mediana inferior no grupo feminino. 0 mesmo ocorreu em relação a peso e altura

O s alunos que apresentavam dor no joeIho foram submetidos a um exame clínicocinésico, para diagnosticar a etiologia da dor e para observar a execução dos padrões de movimento.

\section{RESULTAD OS}

A prevalência de dor no joelho entre os 47 alunos do semestre de 2006.1 foi de $31.9 \%$ (15 alunos). Com um grau de confiança de $95 \%$, podemos afirmar que a verdadeira prevalência de dor no joelho na população estudada está entre $18.6 \%$ a $45.2 \%$. A incidência cumulativa de dor no joelho, numa coorte de 32 alunos, foi acompanhada durante 3 me- ses, com os resultados que se apresentam na Tabela 1.

Tabela 1 - Incidência cumulativa da dor no joelho entre dançarinos

\begin{tabular}{lccc}
\hline Data & $\begin{array}{c}\text { Com Dor } \\
\text { casos novos }\end{array}$ & Sem Dor & $\begin{array}{c}\text { Taxa de Incidência } \\
(\%)\end{array}$ \\
\hline Março/06 & 0 & 32 & $0,0 \%$ \\
Abril/06 & 4 & 28 & 13.3 \\
Maio/06 & 1 & 27 & 2.4 \\
Junho/06 & 1 & 26 & 2.2 \\
\hline
\end{tabular}

\section{DISC USSÃO}

A Escola de $D$ ança da U FBA, mesmo que 0 aluno tenha opção de experimentar técnicas como balé, afro ou dança moderna, éidentificada como uma escola de dança contemporânea, 0 que confere à amostra essa característica.

$O$ s alunos chegam à Escola com um caudal heterogêneo de habilidades técnicas e, em seus corpos, enfrentam o desafio de modificar seus padrões de movimento, para conferir nova significação para suas ações. Padrões de ação fixos de movimentos são, segundo Rodolfo Llinás (2001), "conjuntos de ativações motoras automáticas e bem definidas, que quando se ativam produzem movimentos bem delimitados e coordenados". Para esse autor, a memória e a repetição são dois importantes aspectos no processo de aprendizagem. A repetição de padrões de movimento é mencionada frequentemente pelos professores e pelos dançarinos como uma possibilidade de aprender uma técnica. A repetição de padrões de movimento também é mencionada como um risco no aparecimento de incômodos no joelho. Por outro lado, adquire-se a destreza lentamente, com anos de repetição.

Voltando ao estudo, a freqüência de dor no joelho na amostra, durante o semestre, manteve-se dentro da media esperada na Escola de $D$ ança. $A$ incidência, representada pelos seis novos casos de dor no joelho (Tabela 1), manteve-se entre $13.3 \%$ e $2.2 \%$, apresentando uma gradativa tendência à diminuição. 
0 exame clínico-cinésico permitiu estabelecer duas etiologias para a dor no joel ho nessa amostra. A etiologia mais freqüentemente mencionada da dor do joelho, entre dançarinos, e a síndrome de dor femuro-patelar, ou dor no joelho de origem não específica, que é definida como uma condição de dor peripatelar crônica (REID, 1987). Porém o exame clínico dos seis novos casos permitiu verificar que os traumatismos representados por 3 alunos (50\%) compartilhavam a freqüência dor no joelho com 3 alunos (50\%) com dor de joelho de origem não específica.

O s relatos dos alunos traumatizados por choques contra o chão ou contra companheiros denotavam alguma ineficiência na modulação dos padrões durante as aulas práticas.

Analisou-se também a execução dos padrões de movimento dos membros inferiores nos 3 alunos que apresentavam dor de origem não específica. Repetidamente, foi observado que, quando os padrões dos membros inferiores eram liberados a cada movimento, pensamento ou gesto, os padrões recalcavam-se. Por exemplo, na liberação de padrões de ação fixo, destinados a modular o equilíbrio bi ou uni podal, no contexto da dança ou da marcha, estes evoluíam frequentemente para um padrão que levava repetidamente 0 joelho para a hiper extensão e outras ineficiências.

Esse padrão de ação fixo é muito antigo e representa uma tática no contexto das ações dinâmicas destinadas a manter o corpo na posição vertical. Ele opera autolimitando, no nível do joelho, o equilíbrio anterior do corpo. Essa tática soluciona, através tal padrão de movimento, em muitos indivíduos, os problemas criados pela modulação dos centros de gravidade do corpo e da cabeça em relação aos pés. Esses três dançarinos já apresentavam esse padrão antes de ingressar na Escola.

\section{CONCLUSÕES}

Ambas as etiologias, a traumática e a não específica, que foram reconhecidas entre os dançarinos como possíveis causas de dor no joelho, estariam associadas a ineficiências na modulação dos padrões de ação fixos de movimento ou a solicitações core. $N$ enhuma das etiologias apresentou uma incidência maior sobre a outra, e as atividades, no contexto da dança, não aumentaram sua incidência.

\title{
Candanceprovdeknæpain?
}

\begin{abstract}
Knee pain is one of the most frequent complaints among dancers. The purpose of this study was to establish the prevalence and cumulative incidence of knee pain among dancers to better understand the relation between dance and the dancer's body. Forty-seven students, $n=47$, from the $D$ ance School of the Federal U niversity of Bahia participated in this study. Two methodological designs were chosen for this purpose: a prospective study to establish the prevalence of knee pain, and a cohort study to verify the variation of cumulative incidence of knee pain. The prevalence of knee pain among these dancers was of $31.9 \%$ ( $n=15$ students). The incidence was between $13.3 \%$ and $2.2 \%$, showing a decreasing tendency.
\end{abstract}

Kegwords Dance Knee pain- Incidence.

\section{REFERÊNCIAS}

ASKLIN G, C. et al. A self-reported hamstring injuries in student-dancers, Scand. J. Med. Sci.
Sports, Copenhagen, v.12, n.4, p.230-235, Aug. 2002. 
BOZKURT, $M$. et al. The evaluation of the proximal tibiofibular joint for patients with lateral knee pain. Knee, 0 xford, v.11, n.4, p.307312, Aug. 2004.

BROWN, T.D.; MICHELI, L.J. Foot and ankle injuries in dance. Am. J. O rthop., Chatham, v.33, n.6, p.303-309, June 2004.

CAINE, D.J.; NASSAR, L. Gymnastics injuries. Med. Sport Sci., Basel. v.48, p.18-58, 2005.

CHRIST OFORAKIS, J.J. et al. Internal derangements of the knee associated with patellofemoral joint degeneration. Knee Surg. Sports Traumatol. Arthrosc., H eidelberg, v.13, n.7, p.581-584, 0 ct. 2005.

CIBU LKA, M.T. et al. Patellofemoral pain and asymmetrical hip rotation. Phys. Ther., Alexandria, v.85, n.11, p1201-1207, Nov. 2005.

FREDERICSON, $M$. et al. Physical examination and patellofemoral pain syndrome. Am. J. Phys. Med. Rehabil., H agerstown, v.8, n.3, p.234-243, M ar. 2006.

FREITAS, S.M.; DUARTE, M.; LATASH, M.L. Two kinematic synergies in voluntary whole-body movements during standing. J. Neurophysiol., Bethesda, v.95, n.2, p.636-645, Feb. 2006.

GERBIN O, P.G. et al. Patellofemoral pain syndrome: evaluation of location and intensity of pain. Clin. J. Pain, H agerstown, v.22, n.2, p.154-159, Feb. 2006.

HAM ILTON, D.; ARONSEN, P.; LOKEN, J.H. Dance training intensity at $11-14$ years is associated with femoral torsion in classical ballet dancers. Br. J. Sports M ed., London, v.40, n.4, p.299-303, Apr. 2006.

HILLIER, J.C. et al. Pictorial review: MRI features of foot and ankle injuries in ballet dancers. Br. J. Radiol., London, v.77, n.918, p.532-537, June 2004.

JONSSO N, P.; ALFREDSON, H. Superior results with eccentric compared to concentric quadriceps training in patients with jumper's knee: a prospective randomised study. $\mathbf{B r}$. J. Sports Med., London, v.39, n.11, p.847-850, Nov. 2005.

KHAUND, R.; FLYN N, S.H . Iliotibial band syndrome: a common source of knee pain. Am. Fam. Physician, Kansas City, v.71, n.8, p.15451550, Apr. 2005.

LABELLA, C. Patellofemoral pain syndrome: evaluation and treatment. Prim. C are, Philadel phia, v.31, n.4, p.977-1003. Dec. 2004.

LEITE, C. Eficácia de um programa de treinamento neuromuscular no perfil álgido e na incidência da dor femoropatelar entre dançarinos. 2007. Tese (D outorado em Artes Cênicas) - Escola de Teatro, U niversidade Federal da Bahia, 2007.

LEVINGER, P.; GILLEARD, W. Tibia and rearfoot motion and ground reaction forces in subjects with patellofemoral pain syndrome during walking. Gait Posture, Amsterdam, v.25, n.1, p.2-8, Jan. 2007.

LLINÁS, R.R. El cerebro y el mito del yo. Bogotá: Ed. N orma, 2001.

MIKKELSSON, L.O et al. Adolescent flexibility, endurance strength, and physical activity as predictors of adult tension neck, low back pain, and knee injury: a 25 year follow up study. Br. J. Sports Med., London, v.40, n.2, p.107-113, Feb. 2006.

NAD LER, S.F. et al. Sports and performing arts medicine. 1. General considerations for sports and performing arts medicine. Arch. Phys. Med. Rehabil., Philadelphia, v.85, n.3, p.48-51, M ar. 2004. Suppl. 1.

NEGUS, V.; HOPPER, D.; BRIFFA, N.K. Associations between turnout and lower extremity injuries in classical ballet dancers. J. O rthop. Sports Phys. Ther., Washington, DC, v.35, n.5, p.307-318, M ay 2005.

PARAD OW SKI, P.T. et al. Knee complaints vary with age and gender in the adult population: population-based reference data for the Knee injury and 0 steoarthritis O utcome 
Score (KO O S). BMC M usculoskelet. Disord., London, v.7, p.38, M ay 2006.

PEÑ A, N .; LEITE, C. Prevalencia de lesiones en alumnos de la Escuela de D anza de la Universidad Federal de Bahia-Brasil. Kappa, Buenos Aires, v.2, n.2, p.29-32, 1999.

PEÑ A, N .; LEITE, C. Prevalência e incidência de dor lombar entre dançarinos da Escola de $D$ ança da UFBA. R. Ci. M éd. Biol., Salvador, v.3, n.2, jan./jun. 2004.

POST, W.R. Anterior knee pain: diagnosis and treatment. J. Am. Acad. O rthop. Surg., Rosemont, v.13, n.8, p.534-543, D ec. 2005.

REID, D.C. et al. Lower extremity flexibility patterns in classical ballet dancers and their correlation to lateral hip and knee injuries. Am. J. Sports Med., Thousand 0 aks, v.15, n.4, p.347-352, July/Aug. 1987.

ROUSANOGLOU, E.N .; BOUDOLOS, K.D. $G$ round reaction forces and heart rate profile of aerobic dance instructors during a low and high impact exercise programme. J. Sports Med.
Phys. Fitness., Torino, v.45, n.2, p.162, June 2005.

SH AN , G. Comparison of repetitive movements between ballet dancers and martial artists: risk assessment of muscle overuse injuries and prevention strategies. Res. Sports M ed., Philadelphia, v.13, n.1, p.63-76, Jan./M ar. 2005.

TOLED O, S.D. et al. Sports and performing arts medicine. 6. Issues relating to dancers. Arch. Phys. Med. Rehabil., Philadel phia, v.85, n.3, p.75-78, Mar. 2004. Suppl.1.

WAIN WRIGHT, S.P.; WILLIAMS, C.; TURNER, B. Fractured identities: injury and the ballet body. $\mathrm{H}$ ealth, London, v.9, n.1, p.4966, Jan. 2005.

WATSON, C.J. et al. Reliability and responsiveness of the lower extremity functional scale and the anterior knee pain scale in patients with anterior knee pain. J. O rthop. Sports Phys. Ther., Washintong, DC, v.35, n.3, p.136-146, M ar. 2005. 